\title{
A Solar Power Regulation System Equipped with Intelligent Solar Power Detector
}

\author{
Shun-Yu Chan, Yong-Nong Chang, ${ }^{1 *}$ Hung-Liang Cheng, ${ }^{2}$ and Yih-Her Yan ${ }^{1}$ \\ Department of Electrical Engineering, Cheng Shiu University, Kaohsiung 833, Taiwan, ROC \\ ${ }^{1}$ Department of Electrical Engineering, National Formosa University, Yunlin 632, Taiwan, ROC \\ ${ }^{2}$ Department of Electrical Engineering, I-Shou University, Kaohsiung 840, Taiwan, ROC
}

(Received March 5, 2016; accepted February 27, 2017)

Keywords: solar cell, cubic solar power detector, three-phase power regulation system

This paper describes a solar energy power regulation system using solar power detectors. The system developed comprises a cubic solar power detector, DC-DC converter, three-phase inverter, and a single-chip control module. The cubic solar power detector is composed of five facets installed with front, rear, left, right, and horizontal photo sensors. By detecting solar power coming from different orientations, the solar orientation and solar power can be optimized based on a geometric transformation principle, which evaluates the relationship between photo-power and solar orientation for each sensor. Because the output power of a solar cell can significantly affect the generating capacity of the cell, a solar energy power regulation system was developed to regulate the electricity generated. The solar generating power is monitored by a cubic solar power detector and regulated by a three-phase pulse width modulation inverter. This paper describes the realization and implementation of a solar power regulation system, and experiments have been carried out to validate the performance. Measurements reveal that the system is definitely effective in regulating solar power output and can successfully boost solar power generating capacity.

\section{Introduction}

From the viewpoint of the photovoltaic industry, enhancing conversion efficiency means lowering the cost of generating solar electricity. Recently, among laboratory-developed siliconbased solar cells, the mono-crystalline cell has roughly $25.0 \%$ efficiency; the multi-crystalline cell, 20.4\% efficiency; the copper indium gallium selenide (CIGS) thin film cell, 19.8\% efficiency; and the CdTe thin film cell, 19.6\% efficiency, while the amorphous silicon thin film solar cell can achieve only $10.1 \%$ efficiency. Apart from progress in solar cell fabrication, the alternative and immediate effort is focused on improving the power conversion control and solar panel orientation regulation to promote conversion efficiency in solar electricity generation. ${ }^{(1,2)}$ This is well-known as maximum power point tracking (MPPT) of solar energy control systems.

Considerable literature relating to solar cell MPPT has appeared. According to published principles and realization methodologies, the work can be summarized as the voltage feedback method, the power feedback method, the disturbance observer method, the incremental conductance method, the line approximation method, and the practical measurement method. The associated MPPT methods in literatures are discussed and analyzed as follows:

*Corresponding author: e-mail: ynchang@nfu.edu.tw http://dx.doi.org/10.18494/SAM.2017.1544 
1. Voltage feedback method: ${ }^{(3,4)}$ By achieving a magnitude of MPPT voltage corresponding to certain illumination and temperature in advance, the terminal voltage of a solar cell module may be controlled to match with a predetermined quantity to achieve the MPPT effect. However, two great drawbacks exist, namely, failing to automatically track any additional maximum power point and consuming energy when the atmospheric conditions drastically change, leading to energy loss.

2. Power feedback method: ${ }^{(5,6)}$ Because the voltage feedback method cannot cope with abrupt changes and perhaps fails to reach MPPT, the power feedback method is used which introduces an output power change with respect to a voltage change. When $d P / d V=0$, the MPPT is accomplished. Compared to the voltage feedback method, this method is complicated and requires more computations. However, it can reduce energy loss and enhance total efficiency.

3. Disturbance observer method: ${ }^{(7)}$ In the disturbance observer method, the terminal voltage and output power are changed according to the continuous load variation. By comparing output powers following load variations, the load is adjusted accordingly. When the atmospheric conditions change slowly, the energy consumption worsens. However, due to the simplicity of the method and the few parameters required, the disturbance observer method is still very popular in MPPT applications. Despite that a higher disturbance frequency or disturbance deviation can accelerate the MPPT response, the oscillating problem may be aggravated when the maximum power point is approached. Therefore, the choice of parameters plays a key role in this method.

4. Incremental conductance method: ${ }^{(8,9)}$ The concept of the incremental conductance method is basically similar to that of the power feedback method. It is based on the equation $d P / d V=0$ and can be written as:

$$
\frac{d P}{d V}=\frac{d(V I)}{d V}=I+V \frac{d I}{d V}=0
$$

By rearranging this equation, the following equation can be written:

$$
\frac{d I}{d V}=-\frac{I}{V}
$$

where $d I$ is the current deviation and $d V$ is the voltage deviation. The mismatch of this equation determines the disturbance needed. The MPPT is reached when the incremental conductance $(d I / d V)$ becomes identical with the instantaneous conductance $(I / V)$ of the solar cell with no additional disturbance needed. The incremental conductance method makes MPPT possible under abrupt changes of atmospheric conditions. The major drawbacks include the predetermined $V-I$ characteristics of the solar panel and the need for a larger number of sensing devices, and thereby the requirement for a more complicated circuit to accomplish MPPT.

5. Line approximation method: The line approximation method is the emerging one among MPPT methods. It employs a sliding line to approximate the maximum power point according to various illuminations at a specific temperature. Thus, tracking along the sliding line means realizing MPPT. However, the maximum power point sliding line is established on the basis of a mathematical derivation. The accuracy of various parameters determines the effectiveness of this method. In addition, the aging and deterioration of solar cells and electronic devices probably decrease the precision of this method. 
According to the discussion and analysis of the latest solar tracking methods, merits and drawbacks exist in each method. No superior method exists or is recommended. This paper presents a method to improve the power output of solar cell modules by utilizing solar energy sensors to detect incident solar power. Through a reference table built into a single chip, a threephase inverter is employed to adjust the load. Therefore, the terminal voltage of a solar cell module can be controlled to achieve the maximum power output of a solar cell. Furthermore, the inherited problem of oscillating disturbance losses while approaching the maximum power point resulting from the MPPT methods described can be avoided.

\section{System Structure}

Figure 1 shows the system developed, which comprises a cubic solar power detector, a DC-DC converter, a three-phase inverter, and a single-chip control module. The push-pull type of DC-DC converter is employed to boost the voltage, and closed-loop control is used to stabilize the voltage. In fact, the cubic solar power detector receives photo-power from all orientations and utilizes the single chip module to determine the magnitude of the solar power, thus effectively enhancing the delivered power of the solar cell system.

\section{Principle and Design}

\subsection{DC-DC converter}

As the solar power system operates under conditions of maximum power, the solar cell output voltage is around $30 \mathrm{~V}$. This voltage is not large enough to supply the three-phase inverter with the voltage required by the load. As shown in Fig. 2, the solar cell output voltage is boosted by a DCDC converter, and a voltage closed-loop controller is used to feed the inverter with a voltage of $200 \mathrm{~V}$.

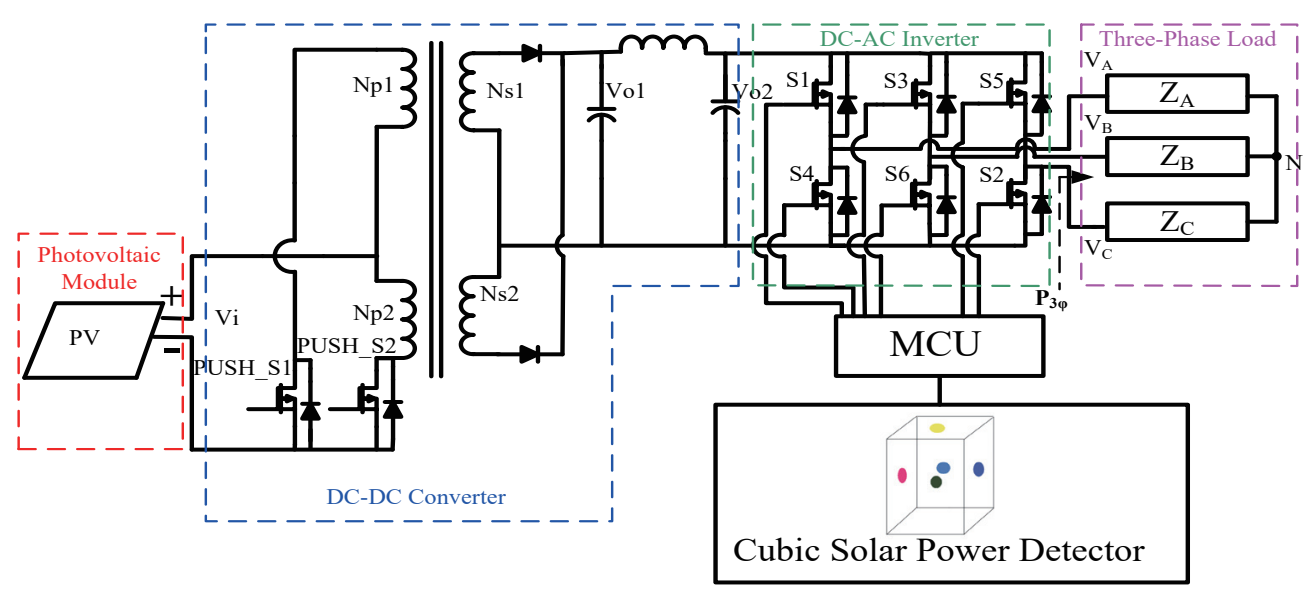

Fig. 1. (Color online) Circuit diagram of the solar power regulation system developed with a solar power detector. 


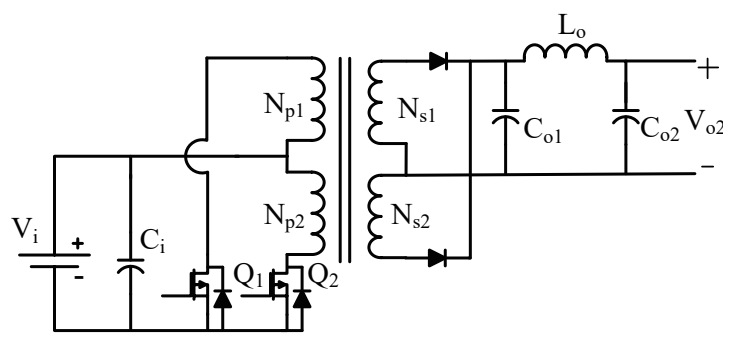

Fig. 2. The proposed push-pull type of DC-DC converter.

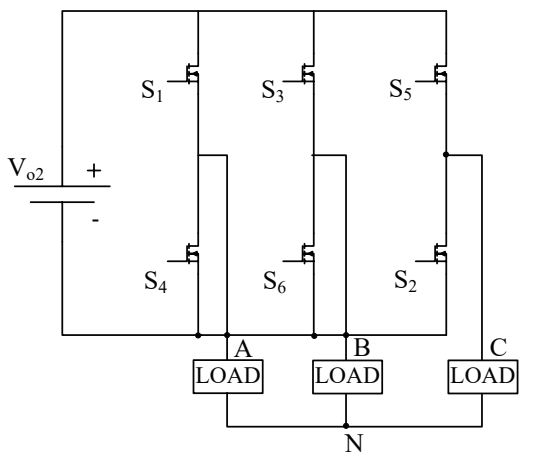

Fig. 3. Diagram of three-phase inverter.

\subsection{Three-phase inverter}

Figure 3 shows the circuit structure of a three-phase inverter. In this solar power system, the six-step square-wave mode is used with three phase legs of inverter-generating square waves at a mutual phase displacement angle of $120^{\circ}$. In addition, the PWM technique is introduced into the modulating scheme as well. Therefore, the three-phase inverter modulates the duty-cycle according to the solar power absorbed and then regulates the output power on the load.

\subsection{Cubic solar power detector}

The cubic solar power detector is composed of five facets installed with front, rear, left, right, and horizontal photosensors. Coming from different orientations, the incident sunlight can be decomposed into four quadrants. As shown in Fig. 4, five sensors are placed on horizontal, right, left, rear, and front facets, respectively, where the front, right, and horizontal sensors can absorb incident sunlight coming from the first quadrant; the front, left, and horizontal sensors can absorb incident sunlight coming from the second quadrant; the rear, left, and horizontal sensors can absorb incident sunlight coming from the third quadrant, and the rear, right, and horizontal sensors can absorb incident sunlight coming from the fourth quadrant.

To take one corner of the cubic solar power detector as the origin of the Cartesian coordinate system, the position of the light source is located at coordinate $(X, Y, Z)$. As shown and defined in Fig. 5, the distance between the horizontal sensor and the sun is $r$, the incident angle of sunlight is $\phi$, and the orientation angle is $\alpha$.

Based on coordinate transformation, the three-dimension parameters can be expressed as Eq. (1):

$$
\text { The coordinate of lighting source }\left\{\begin{array}{l}
x=r \sin \phi \cos \alpha \\
y=r \sin \phi \sin \alpha, \\
z=r \cos \phi
\end{array}\right. \text {, }
$$

where $\phi$ is the angle between the normal line (Z-axis) of the horizontal facet and incident sunlight, $\alpha$ is the angle between $X$-axis and the projection of the sun on $X Y$ plane, and $r$ is the distance between the sun and the origin. 


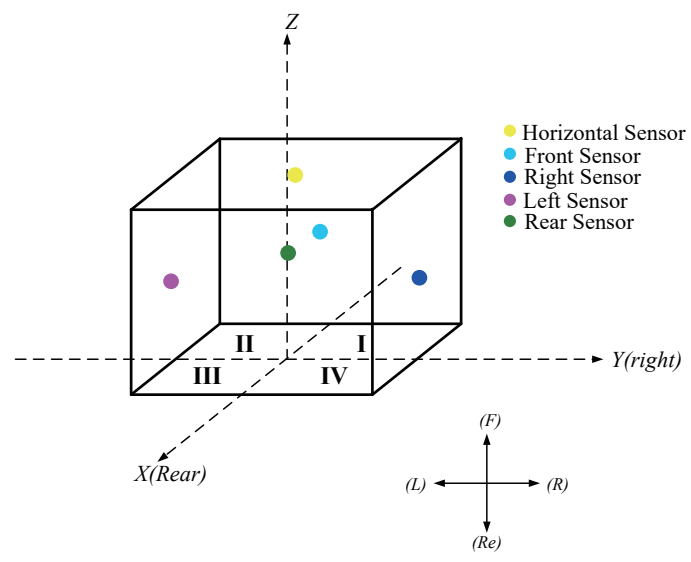

Fig. 4. (Color online) Schematic model of cubic solar power detector.

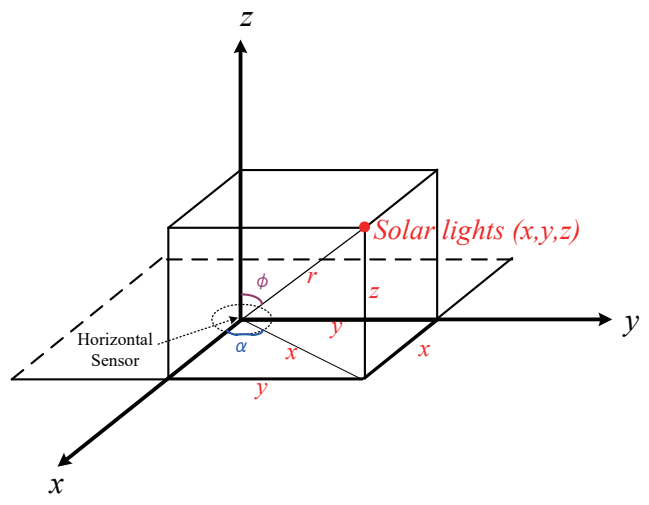

Fig. 5. (Color online) Definition of parameters in space.

Indeed, the incident angles of sunlight are different for sensors located at different facets. Since the defined parameters are based on the horizontal sensor, the incident power on the horizontal sensor can be described by Eq. (2), wherein $P_{D}$ is the measured upright direct incident power. Thus, the incident power on the horizontal sensor is proportional to $\cos \phi$.

$$
P_{H D}=P_{D} \cos \phi
$$

For sensors located on different facets, the corresponding coordinates for different sensors can be achieved based on coordinate transformation. ${ }^{(10)}$ To describe the absorption of solar power by each sensor, many mathematical derivations must be made, and they reveal the power calculations on the five sensors. By referring to the coordinate correspondence expressed in Eq. (1) and the definitions of incident horizontal angle $\phi$ and vertical angle $\alpha$, the absorption power of the sensors on the five facets of a cubic detector can be derived accordingly.

For the horizontal sensor, the sunlight incident angle $\phi_{H}$ is equal to $\phi$. The measured solar power on the horizontal sensor includes the upright direct incident power $P_{D}$ and the horizontal scattering and diffuse solar power $P_{s k y H}$. Thus, the relationship between the measured solar power on horizontal sensor $P_{H}$ and the upright maximum power $P_{D}$ can be written as Eq. (3).

$$
P_{H}=P_{D} \cos \phi+P_{s k y H}=P_{D} \cos \phi_{H}+P_{s k y H}
$$

Similarly, the incident angle of sunlight on the right-facet sensor is equal to $\phi_{R}$.

$$
\begin{gathered}
\phi_{R}=\cos ^{-1}(\sin \phi \sin \alpha) \\
P_{R}=P_{D} \sin \phi \sin \alpha+P_{s k y H}=P_{D} \cos \phi_{R}+P_{s k y R}
\end{gathered}
$$

Likewise, the incident angle of sunlight on the left-facet sensor is equal to $\phi_{L}$, and Eq. (5) can be written as 


$$
\begin{gathered}
\phi_{L}=\cos ^{-1}(-\sin \phi \sin \alpha), \\
P_{L}=-P_{D} \sin \phi \sin \alpha+P_{s k y L}=P_{D} \cos \phi_{L}+P_{s k y L} .
\end{gathered}
$$

By denoting the incident angle of sunlight on the front sensor as $\phi_{F}$, Eq. (6) can be derived.

$$
\begin{gathered}
\phi_{F}=\cos ^{-1}(-\sin \phi \sin \alpha) \\
P_{F}=-P_{D} \sin \phi \cos \alpha+P_{s k y F}=P_{D} \cos \phi_{F}+P_{s k y F}
\end{gathered}
$$

By designating the incident angle of sunlight on the rear-facet sensor as $\phi_{R e}$, Eq. (7) can be written as

$$
\begin{gathered}
\phi_{R e}=\cos ^{-1}(\sin \phi \cos \alpha), \\
P_{R e}=P_{D} \sin \phi \cos \alpha+P_{s k y R e}=P_{D} \cos \phi_{R e}+P_{s k y R e} .
\end{gathered}
$$

Successively, by using Eqs. (3)-(7), the parameters $\phi, \alpha$, and $P_{D}$ can be obtained as follows.

$$
\begin{gathered}
P_{F D}=P_{D} \cos \phi_{F}=P_{F}-P_{s k y F}=P_{D} \sin \phi \cos \alpha \\
P_{R D}=P_{D} \cos \phi_{R}=P_{R}-P_{s k y R}=P_{D} \sin \phi \sin \alpha \\
\frac{P_{R D}}{P_{F D}}=\frac{P_{D} \sin \phi \sin \alpha}{P_{D} \sin \phi \cos \alpha}=\frac{\sin \alpha}{\cos \alpha}=\tan \alpha \Rightarrow \alpha=\tan ^{-1}\left(\frac{P_{R D}}{P_{F D}}\right)
\end{gathered}
$$

According to Eqs. (3) and (6), the incident horizontal angle $\phi$ is given as

$$
\begin{gathered}
P_{H D}=P_{D} \cos \phi_{H}=P_{H}-P_{s k y H}=P_{D} \cos \alpha \\
\frac{P_{F D}}{P_{H D}}=\frac{-\sin \phi \sin \alpha}{\cos \phi}=-\tan \phi \cos \alpha \Rightarrow \phi=\tan ^{-1}\left(\frac{P_{F D}}{P_{H D} \cos \alpha}\right) .
\end{gathered}
$$

Next, by substituting $\phi$ into Eq. (2), the upright direct incident power $P_{D}$ can be determined using

$$
P_{D}=\frac{P_{H D}}{\cos \phi}
$$

\section{Experimental Results and Discussion}

With further reference to Fig. 1 showing the solar power regulation system, the control stage is made up of two power converter stages and different solar power detectors. The front power stage is used for stabilizing the dc link voltage, while the second power stage is employed for power regulation. In cases of different solar power inputs, the practical measurements are carried out to validate the availability of the solar power regulation system. 
Figure 6 shows the waveforms of the solar power regulation system in the case of $865 \mathrm{~W} / \mathrm{m}^{2}$ solar power. Figure 6(a) displays the waveforms of a push-pull converter. By regulating the duty cycle of the trigger signals $P U S H_{-} V_{g s 1}$ and $P U S H_{-} V_{g s 2}$, the dc link voltage $V_{o 2}$ can be controlled and stabilized, thus providing the inverter with a steady dc voltage. Figure 6(b) shows the waveforms of the inverter output power and three phase voltages on the load. It is obvious that the voltage waveforms show $120^{\circ}$ duration due to the six-step square wave method being used.

Figure 7 illustrates the waveforms of the solar power regulation system with a solar detector when the solar power is $572 \mathrm{~W} / \mathrm{m}^{2}$. Even under the same solar power, the solar cell can supply the corresponding power according to the load change. This research utilized a single chip to regulate the output power by controlling the duty cycle of power switch. Figure 7(a) shows voltage waveforms and power waveforms when the solar power is $572 \mathrm{~W} / \mathrm{m}^{2}$. The push-pull converter can stabilize the dc link voltage and supply the three-phase inverter with a stable dc voltage. By inspecting Fig. 7(b), it can be clearly seen that the inverter output power is smaller than that shown in Fig. 6(b).

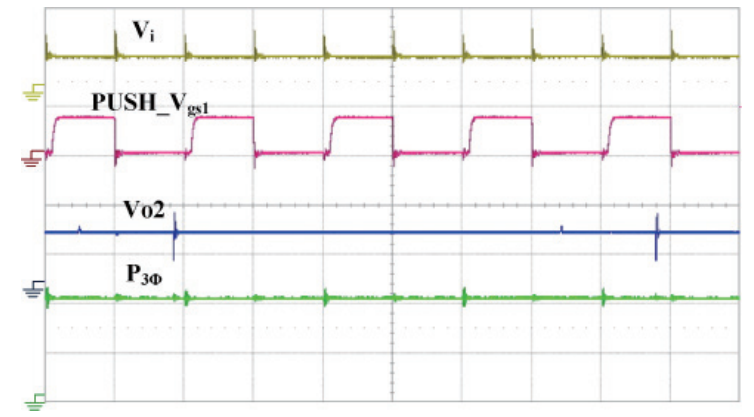

(a)

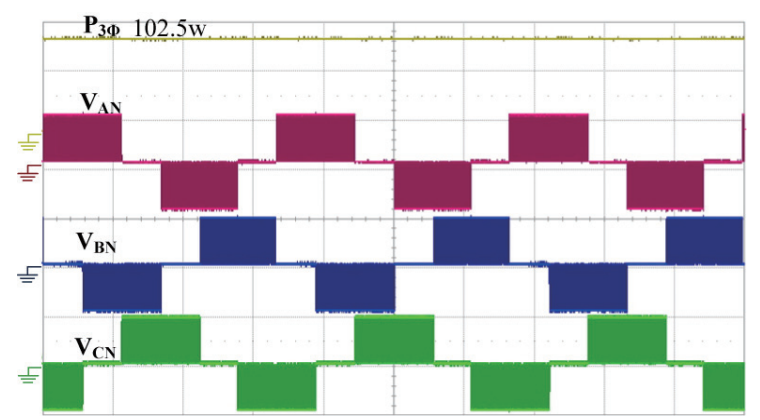

(b)

Fig. 6. (Color online) Waveforms of the solar power regulation system in the case of $865 \mathrm{~W} / \mathrm{m}^{2}$ solar power. (a) The waveforms of the push-pull DC-DC converter $\left(V_{i}: 50 \mathrm{~V} /\right.$ div; $P U S H_{-} V_{g s 1}: 20 \mathrm{~V} / \mathrm{div} ; V_{o 2}: 200 \mathrm{~V} / \mathrm{div} ; P_{3 \Phi}: 50$ W/div; Time: $10 \mu \mathrm{s} / \mathrm{div})$; (b) The waveforms of the inverter $\left(P_{3 \Phi}: 50 \mathrm{~W} / \mathrm{div} ; V_{A N}: 100 \mathrm{~V} / \mathrm{div} ; V_{B N}: 100 \mathrm{~V} / \mathrm{div} ; V_{C N}\right.$ : $100 \mathrm{~V} /$ div; Time: $5 \mathrm{~ms} / \mathrm{div})$.

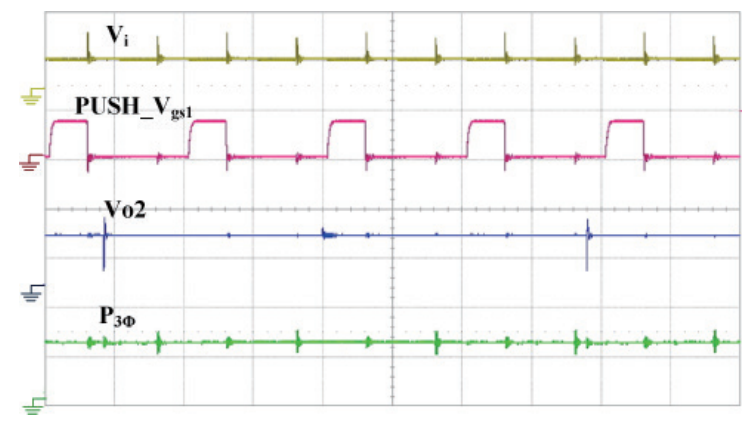

(a)

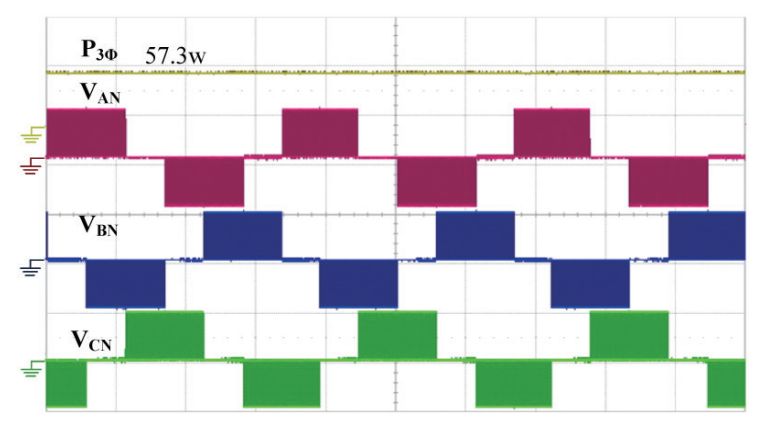

(b)

Fig. 7. (Color online) Waveforms of the solar power regulation system in the case of $572 \mathrm{~W} / \mathrm{m}^{2}$ solar power. (a) The waveforms of the push-pull DC-DC converter $\left(V_{i}: 50 \mathrm{~V} /\right.$ div; $P U S H_{-} V_{g s 1}: 20 \mathrm{~V} /$ div; $V_{o 2}: 200 \mathrm{~V} /$ div; $P_{3 \Phi}: 50$ $\mathrm{W} /$ div; Time: $10 \mu \mathrm{s} / \mathrm{div})$; (b) The waveforms of the inverter $\left(P_{3 \Phi}: 50 \mathrm{~W} / \mathrm{div} ; V_{A N}: 100 \mathrm{~V} / \mathrm{div} ; V_{B N}: 100 \mathrm{~V} / \mathrm{div} ; V_{C N}\right.$ : $100 \mathrm{~V} /$ div; Time: $5 \mathrm{~ms} / \mathrm{div})$. 


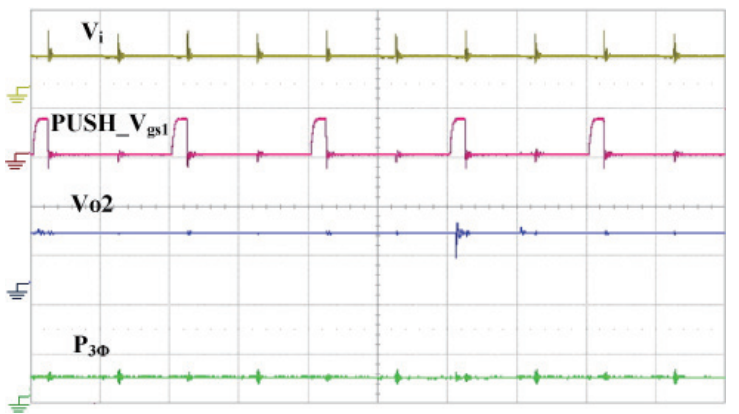

(a)

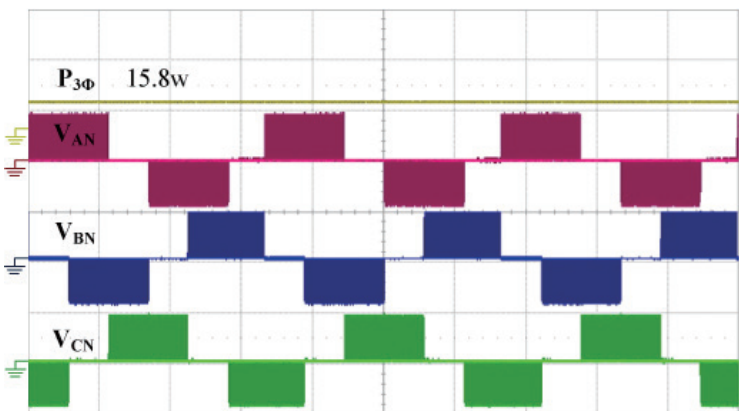

(b)

Fig. 8. (Color online) Waveforms of the solar power regulation system in the case of $182 \mathrm{~W} / \mathrm{m}^{2}$ solar power. (a) The waveforms of the push-pull DC-DC converter $\left(V_{i}: 50 \mathrm{~V} /\right.$ div; $P U S H_{-} V_{g s 1}: 20 \mathrm{~V} /$ div; $V_{o 2}: 200 \mathrm{~V} /$ div; $P_{3 \Phi}: 50$ $\mathrm{W} /$ div; Time: $10 \mu \mathrm{s} / \mathrm{div})$; (b) The waveforms of the inverter $\left(P_{3 \Phi}: 50 \mathrm{~W} / \overline{\mathrm{div}} ; V_{A N}: 100 \mathrm{~V} / \mathrm{div} ; V_{B N}: 100 \mathrm{~V} / \mathrm{div} ; V_{C N}\right.$ : $100 \mathrm{~V} /$ div; Time: $5 \mathrm{~ms} / \mathrm{div})$.

Figure 8 shows the waveforms of the solar power regulation system in the case of $182 \mathrm{~W} / \mathrm{m}^{2}$ solar power. Figure 8(a) displays the waveforms of the push-pull converter. The push-pull converter can stabilize the de link voltage and feed inverter with a steady de voltage. Figure 8(b) shows that the inverter output power is smaller than those shown in Figs. 6(b) and 7(b).

\section{Conclusions}

This research integrates a cubic solar power detector, a DC-DC converter, a three-phase inverter, and a single-chip control module to achieve a solar energy power regulation system with a solar orientation tracker. The system developed is successful at approaching the maximum power point and can effectively increase the generating capacity of solar cell modules. A practical solar energy power regulation system was implemented and measured to validate the performance and availability of this three-phase energy regulating system. According to the experimental results, the structure developed for the solar energy power regulation system is effective in regulating the output power and enhancing the generating power of a solar cell module.

\section{References}

1 K. B. Shah and L. P. Joshi: Nirma University Int. Conf. Engineering (2013) pp.1-6.

2 S. K. Kollimalla and M. K. Mishra: Nirma University Int. Conf. Engineering (2013) pp. 653-658.

3 N. Adhikari, B. Singh, and A. L. Vyas: IEEE Int. Conf. Industrial and Information Systems (2012) pp.1-6.

4 H. Patel and V. Agarwal: IEEE Trans. Ener. Conversion 24 (2009) 256.

5 A. K. Abdelsalam, A. M. Massoud, S. Ahmed, and P. Enjeti: IEEE Trans. Power Electron. 26 (2011) 1010.

6 G. Carannante, C. Fraddanno, M. Pagano, and L. Piegari: IEEE Trans. Ind. Electron. 56 (2009) 4374.

7 D. Sera, L. Mathe, T. Kerekes, S. V. Spataru, and R. Teodorescu: J. Photovoltaics 3 (2013) 1070

8 C. S. Murdoch and S. N. Reynoso: IEEE Lat. Am. Trans. 11 (2013) 108.

9 A. Nabulsi and R. Dhaouadi: IEEE Trans. Ind. Inf. 8 (2012) 573.

10 Y. N. Chang and H. L. Cheng: Sens. Mater. 26 (2014) 365 


\section{About the Authors}
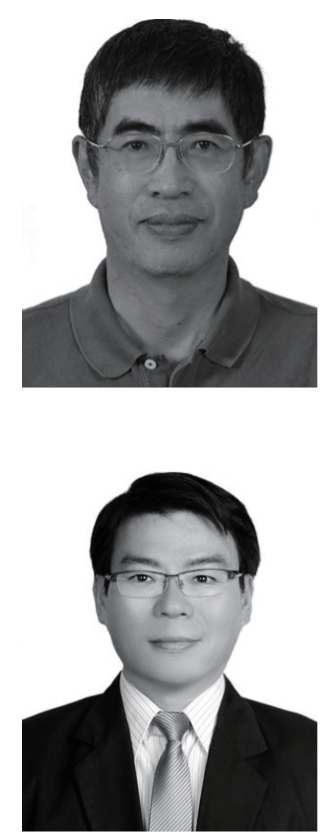

Shun-Yu Chan was born in Kaohsiung, Taiwan, in 1959. He received a B.S. degree in electrical engineering from the National Tsing Hua University, Hsinchu, Taiwan, in 1982, and M.S. and Ph.D. degrees in electrical engineering from the National Sun Yat-Sen University, Kaohsiung, in 1987 and 1998, respectively. He is currently an associate professor in the Department of Electrical Engineering, Cheng Shiu University, Kaohsiung. His research interests include lighting electronics and power system applications.

Yong-Nong Chang was born in Yunlin, Taiwan, in 1964. He received B.S., M.S., and Ph.D. degrees in electrical engineering from the National Sun YatSen University, Kaohsiung, Taiwan, in 1986, 1988, and 1995, respectively. Since 1995, he has been with National Formosa University, Yunlin, where he is currently a professor in the Department of Electrical Engineering. His current research interests include power electronics and their applications, particularly in lighting electronics and battery systems.

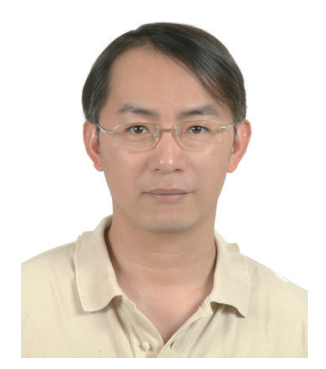

Hung-Liang Cheng was born in Chunghwa, Taiwan, in 1964. He received B.S., M.S., and Ph.D. degrees in electrical engineering from the National Sun Yat-Sen University, Kaohsiung, Taiwan, in 1986, 1988, and 2001, respectively. From 1988 to 2007, he was an electronic researcher with the Chung-Shan Institute of Science and Technology, Taoyuan County, Taiwan, where he designed and developed high-power transmitters in radar and missile systems. Since February 2007, he has been with the Department of Electrical Engineering, I-Shou University, Kaohsiung, where he is currently a professor. His current research interests include power-electronic converters and electronic ballasts/drivers for lighting applications.

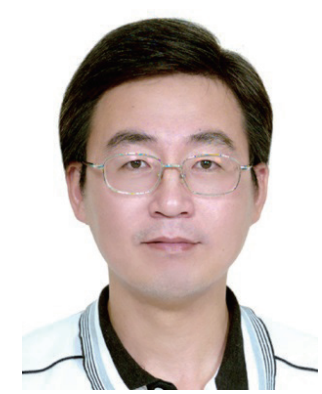

Yih-Her Yan was born in Kaohsiung, Taiwan, Republic of China, in 1964. He received B.S., M.S., and Ph.D. degrees in electrical engineering from National Sun Yat-Sen University in 1986, 1988, and 1992, respectively. He now teaches at National Formosa University, Yunlin, Taiwan. His research interests are in the areas of power system analysis, harmonic analysis, and industrial applications of power electronics. 